\title{
Front Matter: Volume 11124
}

, "Front Matter: Volume 11124," Proc. SPIE 11124, Terahertz Emitters, Receivers, and Applications X, 1112401 (14 October 2019); doi:

10.1117/12.2551552

EDIE Event: SPIE Optical Engineering + Applications, 2019, San Diego, California, SPIE. United States 


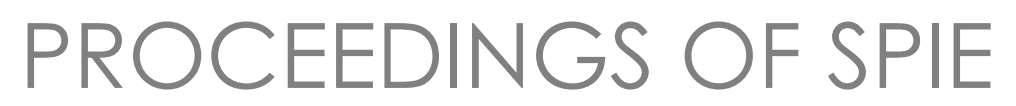

\title{
Terahertz Emitters, Receivers, and Applications $X$
}

\author{
Manijeh Razeghi \\ Alexei N. Baranov \\ Miriam S. Vitiello \\ Editors
}

11-13 August 2019

San Diego, California, United States

Sponsored and Published by

SPIE

Volume 11124 
The papers in this volume were part of the technical conference cited on the cover and title page. Papers were selected and subject to review by the editors and conference program committee. Some conference presentations may not be available for publication. Additional papers and presentation recordings may be available online in the SPIE Digital Library at SPIEDigitalLibrary.org.

The papers reflect the work and thoughts of the authors and are published herein as submitted. The publisher is not responsible for the validity of the information or for any outcomes resulting from reliance thereon.

Please use the following format to cite material from these proceedings:

Author(s), "Title of Paper," in Terahertz Emitters, Receivers, and Applications X, edited by Manijeh Razeghi, Alexei N. Baranov, Miriam S. Vitiello, Proceedings of SPIE Vol. 11124 (SPIE, Bellingham, WA, 2019) Seven-digit Article CID Number.

ISSN: 0277-786X

ISSN: 1996-756X (electronic)

ISBN: 9781510629417

ISBN: 9781510629424 (electronic)

Published by

SPIE

P.O. Box 10, Bellingham, Washington 98227-0010 USA

Telephone +1 3606763290 (Pacific Time) · Fax +1 3606471445

SPIE.org

Copyright (c) 2019, Society of Photo-Optical Instrumentation Engineers.

Copying of material in this book for internal or personal use, or for the internal or personal use of specific clients, beyond the fair use provisions granted by the U.S. Copyright Law is authorized by SPIE subject to payment of copying fees. The Transactional Reporting Service base fee for this volume is $\$ 21.00$ per article (or portion thereof), which should be paid directly to the Copyright Clearance Center (CCC), 222 Rosewood Drive, Danvers, MA 01923. Payment may also be made electronically through CCC Online at copyright.com. Other copying for republication, resale, advertising or promotion, or any form of systematic or multiple reproduction of any material in this book is prohibited except with permission in writing from the publisher. The CCC fee code is 0277$786 \times / 19 / \$ 21.00$.

Printed in the United States of America by Curran Associates, Inc., under license from SPIE.

Publication of record for individual papers is online in the SPIE Digital Library.

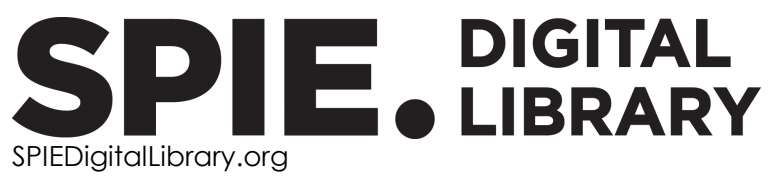

Paper Numbering: Proceedings of SPIE follow an e-First publication model. A unique citation identifier (CID) number is assigned to each article at the time of publication. Utilization of CIDs allows articles to be fully citable as soon as they are published online, and connects the same identifier to all online and print versions of the publication. SPIE uses a seven-digit CID article numbering system structured as follows:

- The first five digits correspond to the SPIE volume number.

- The last two digits indicate publication order within the volume using a Base 36 numbering system employing both numerals and letters. These two-number sets start with $00,01,02,03,04$, 05, 06, 07, 08, 09, 0A, OB ... 0Z, followed by 10-1Z, 20-2Z, etc. The CID Number appears on each page of the manuscript. 


\section{Contents}

$\begin{array}{cl}\vee & \text { Authors } \\ \text { vii } & \text { Conference Committee }\end{array}$

THZ QUANTUM CASCADE LASERS

$1112404 \quad$ Aperiodic photonic architectures for high-power distributed feedback THz quantum cascade lasers (Invited Paper) [1 $11124-2]$

1112406 Graphene-based van der Waals heterostructures towards a new type of terahertz quantum-cascade laser (Invited Paper) [1 $11124-4]$

NOVEL CONCEPTS AND MATERIALS FOR THZ TECHNOLOGY I

$1112407 \quad$ Room temperature continuous wave THz frequency comb based on quantum cascade lasers (Keynote Paper) [1 $11124-5]$

1112409 Spectral properties of incoherent terahertz torch based on parabolic Ga(As,Bi)/AIGaAs quantum wells [1 $1124-7]$

\section{SOURCES OF THZ RADIATION}

$11124 \mathrm{OB} \quad$ Vortex terahertz wave generation in air by femtosecond optical vortex pulses [1 1124-9]

$11124 \mathrm{OD} \quad$ Upscaling the output power of a photo-mixing $\mathrm{THz}$ source driven by a dual-frequency laser operating on two transverse modes (Invited Paper) [11124-11]

FUNDAMENTALS OF GENERATION, DETECTION, AND PROPAGATION OF THZ WAVES

$111240 G$ Towards realisation of an efficient continuous wave terahertz source using quantum dot devices [1 $1124-15]$

\section{THZ SPECTROSCOPY}

11124 ON Terahertz frequency metrology (Invited Paper) [11124-22]

1112400 The THz sum-frequency counterparts of stimulated Raman scattering (Invited Paper) [1 $1124-23]$ 
NOVEL CONCEPTS AND MATERIALS FOR THZ TECHNOLOGY II

11124 OP Chip-scale terahertz systems (Invited Paper) [11124-24]

$111240 Q \quad$ Broadband terahertz gas spectroscopy through multimode self-mixing in a quantum cascade laser (Invited Paper) [1 $11124-25]$

$11124 \mathrm{OR} \quad \mathrm{Pb}_{1-\mathrm{x}} \mathrm{Sn}_{\mathrm{x}} \mathrm{Se}$ : a new tunable topological platform with terahertz band gap (Invited Paper) [11 1 124-26]

11124 OS Terahertz waveguide signal processing: passive and active devices (Invited Paper) [11124-27]

IMAGING

$111240 \mathrm{OU} \quad$ Terahertz-wave radars based on resonant-tunneling-diode oscillators (Invited Paper) [1 $11124-30]$

11124 OW Terahertz spatial light modulator with more than 1 THz working range (Invited Paper) [11124-32]

THZ DETECTORS

$111240 X \quad$ Fibonacci subterahertz imaging: features and applications [11124-33]

1112410 Terahertz detectors based on all-dielectric photoconductive metasurfaces (Invited Paper) [11 1124-36]

\section{POSTER SESSION}

$1112411 \quad$ Fourier imaging with CW terahertz waves [11124-29]

1112412 External electric field control of terahertz radiation from laser-induced air plasma [11124-37]

1112415 Large field-of-view continuous-wave terahertz reflective off-axis digital holography [11124-41]

1112416 A two-phase flow meter targeting high GVF [11124-42]

iv 


\section{Authors}

Numbers in the index correspond to the last two digits of the seven-digit citation identifier (CID) article numbering system used in Proceedings of SPIE. The first five digits reflect the volume number. Base 36 numbering is employed for the last two digits and indicates the order of articles within the volume. Numbers start with 00, 01, 02, 03, 04, 05, 06, 07, 08, 09, 0A, 0B...0Z, followed by 10-12, 20-2Z, etc.

\author{
Abbes, Alaeddine, OD \\ Al Amri, Abdelaziz, 16 \\ Al Bulooshi, Qasim, 16 \\ Al Shehi, Mahmoud, 16 \\ Asada, Masahiro, OU \\ Assaf, B. A., OR \\ Auriacombe, $O ., 0 Q$ \\ Bartalini, Saverio, ON \\ Bastard, G., OR \\ Baver, G., OR \\ Beaudoin, Grégoire, OD \\ Beere, Harvey E., 04 \\ Bello, Nasir G., OG \\ Berge, LuC, OB \\ Biasco, Simone, 04 \\ Blin, Stéphane, OD \\ Boubanga Tombet, Stephane, 06 \\ Brener, Igal, 10 \\ Brewster, N., OQ \\ Buožius, Danas, $\mathrm{OB}$ \\ Butkute, Renata, 09 \\ Cappelli, Francesco, ON \\ Chhantyal-Pun, R., OQ \\ Chomet, Baptiste, OD \\ Consolino, Luigi, $\mathrm{ON}$ \\ Davies, A. Giles, 04, 0Q \\ Dean, $P ., O Q$ \\ De Natale, Paolo, $0 \mathrm{~N}$ \\ De Regis, Michele, ON \\ de Vaulchier, L.-A., OR \\ Devenson, Jan, 09 \\ Dickey, Michael D., OS \\ Dobroiu, Adrian, OU \\ Dong, Liquan, 12 \\ Dong, R., OQ \\ Dubinov, Alexander A., 06 \\ Ellison, B. N., OQ \\ Feng, Shijia, 12 \\ Ferreira, R., OR \\ Garnache, Arnaud, OD \\ Garrasi, Katia, 04 \\ Gorodetsky, Andrei, OG \\ Guldner, Y., OR \\ Hale, Lucy, 10 \\ Han, Y. J., OQ \\ Harris, Charles Thomas, 10 \\ Henry, M., OQ \\ Indrišiūnas, Simonas, $\mathrm{OX}$ \\ Ivanov, Maksym, OB
}

Jakštas, Vytautas, 09

Jokubauskis, Domas, OX

Joshipura, Ishan D., OS

Juraschek, Dominik M., 00

Kampfrath, Tobias, 00

Kappa, J., OW

Karaliūnas, Mindaugas, 09, 0X

Kašalynas, Irmantas, OX

Keeley, J., OQ

Klingel, S., OW

Krizman, G., OR

Li, Fen, 11

Li, Lianhe, 04, OQ

Linfield, Edmund H., 04, OQ

Lisauskas, Alvydas, 11

Lozada-Smith, Nicolas, OS

LU, Q. Y., 07

LUk, Ting Shan, 10

Ma, Jianjun, OS

Maehrlein, Sebastian F., 00

Mendis, Rajind, OS

Minkevičius, Linas, OX

Mitrofanov, Oleg, 10

Mittleman, Daniel M., OS

Myara, Mikhaël, OD

Nafa, Malik, ON

Norkus, Ričardas, 09

Nuttall, E., $O Q$

Oesterschulze, E., OW

Oldfield, M., $0 Q$

Otsuji, Taiichi, 06

Paarmann, Alexander, 00

Pagalys, Justas, 09

Partington, J., $0 Q$

Račiukaitis, Gediminas, OX

Rafailov, Edik U., OG

Rahm, M., OW

Rawlings, T., OQ

Razeghi, M., 07

Reichel, Kimberly S., OS

Reno, John L., 10

Ritchie, David A., 04

Rong, LU, 15

Roskos, Hartmut G., 11

Ryzhii, Victor, 06

Sagnes, Isabelle, OD

Satou, Akira, 06

Sengupta, Kaushik, OP

Shemelya, C., OW 
Shrestha, Rabi, OS

Siday, Thomas, 10

Skupin, Stefan, OB

Slivken, S., 07

Smirnov, Semen, OG

Sokoluk, D., OW

Springholz, G., OR

Suzuki, Safumi, OU

Thiele, Illia, OB

Udal, Andres, 09

Urbanowicz, Andrzej, 09

Vabishchevich, Polina P., 10

Vaičaitis, Virgilijus, $O B$

Valavanis, A., OQ

Valušis, Gintaras, 09, 0X

Vitiello, Miriam S., 04

Voß, Daniel, 11

Wang, Dayong, 15

Wang, F. H., 07

Watanabe, Takayuki, 06

Wu, D. H., 07

Yadav, Deepika, 06

Yu, Aoxue, 12

Yuan, Hui, 11

Zhang, Liangliang, 12

Zhao, Yuejin, 12

Proc. of SPIE Vol. 11124 1112401-6

Downloaded From: https://www.spiedigitallibrary.org/conference-proceedings-of-spie on 25 Apr 2023 Terms of Use: https://www.spiedigitallibrary.org/terms-of-use 


\title{
Conference Committee
}

\author{
Program Track Chair
}

Ruyan Guo, The University of Texas at San Antonio (United States)

\section{Conference Chairs}

Manijeh Razeghi, Northwestern University (United States)

Alexei N. Baranov, Université Montpellier (France)

Miriam S. Vitiello, Consiglio Nazionale delle Ricerche (Italy)

\section{Conference Program Committee}

Stefano Barbieri, IEMN, Université Lille 1 (France)

Robert J. Grasso, Polaris Alpha (United States)

Sven Höfling, Julius-Maximilians-Universität Würzburg (Germany)

Hiroshi Ito, Kitasato University (Japan)

Juliette Mangeney, Laboratoire Pierre Aigrain (France)

Oleg Mitrofanov, University College London (United Kingdom)

Gaël Mouret, Université du Littoral Côte d'Opale (France)

Mauro F. Pereira, Institute of Physics of the CAS, v.v.i. (Czech Republic)

Rohit P. Prasankumar, Los Alamos National Laboratory (United States)

Edik U. Rafailov, Aston University (United Kingdom)

Roland Teissier, Université Montpellier (France)

Frédéric Teppe, Université Montpellier (France)

Dmitry Turchinovich, Universität Duisburg-Essen (Germany)

Vladimir V. Vaks, Institute of Applied Physics of the RAS

(Russian Federation)

Gintaras Valušis, Center for Physical Sciences and Technology (Lithuania)

Session Chairs

1 THz Quantum Cascade Lasers

Alexei N. Baranov, Université Montpellier (France)

Gintaras Valušis, Center for Physical Sciences and Technology (Lithuania)

2 Novel Concepts and Materials for THz Technology I

Sushil Kumar, Lehigh University (United States)

Dmitry Turchinovich, Universität Bielefeld (Germany)

3 Sources of $\mathrm{THz}$ Radiation

Alvydas Lisauskas, Vilnius University (Lithuania)

Nicholas Sirica, Los Alamos National Laboratory (United States) 
4 Fundamentals of Generation, Detection, and Propagation of $\mathrm{THz}$ Waves Marco Rahm, Technische Universität Kaiserslautern (Germany) Stefan Kaiser, Max-Planck-Institut für Festkörperforschung (Germany)

5 THz Spectroscopy

Rolando Valdes Aguilar, The Ohio State University (United States)

Kaushik Sengupta, Princeton University (United States)

6 Novel Concepts and Materials for THz Technology II

Oleg Mitrofanov, University College London (United Kingdom)

Paola Di Pietro, Elettra-Sincrotrone Trieste S.C.p.A. (Italy)

7 Imaging

Martin Mittendorff, Universität Duisburg-Essen (Germany)

Joshua Freeman, University of Leeds (United Kingdom)

$8 \mathrm{THz}$ Detectors

Alexei N. Baranov, Université Montpellier (France)

Joshua Freeman, University of Leeds (United Kingdom) 